\title{
Diagnosis and treatment of gastric duplication in children: A case report
}

\author{
LIFENG ZHANG, QINGJIANG CHEN, ZHIGANG GAO, QIXING XIONG and QIANG SHU \\ Department of Endoscopic Surgery Center, Children's Hospital, Zhejiang University School of Medicine, \\ Hangzhou, Zhejiang 310000, P.R. China
}

Received January 29, 2016; Accepted May 15, 2017

DOI: $10.3892 /$ etm.2017.4895

\begin{abstract}
Gastric duplication is a rare congenital abnormality, and its diagnosis and treatment may be challenging in certain children. The present study reported four cases of gastric duplication who were followed-up and treated in the Children's Hospital of Zhejiang University between March 2010 and March 2015. The study aimed to increase the knowledge on this disease in order to improve the diagnosis and treatment, as well as decrease the possibility of misdiagnosis. Diagnosis could not be confirmed by the clinical manifestation, ultrasonography and computed tomography (CT). Therefore, laparoscopic examination and laparotomy were conducted, which confirmed the gastric duplication, and resection was performed according to the type of gastric duplication. Two patients received laparoscopy-assisted resection by removing the duplicated gastric tube through the navel, while one patient received total laparoscopic resection, and one patient received laparoscopy and laparotomy. The surgical procedures were performed without any issues, and no complications appeared following the surgery. The preoperative symptoms disappeared completely, and the patients recovered well according to the follow-ups conducted at 1 month, 1 year and 3 years for all four cases. In conclusion, gastric duplication is a rare condition and its preoperative diagnosis is difficult, and laparotomy is an effective method for diagnosis. Laparoscopy-assisted resection or total laparoscopic resection is preferred for uncomplicated gastric duplications, and simple excision can achieve a good therapeutic effect.
\end{abstract}

\section{Introduction}

Gastric duplication is a rate type of congenital abnormality with an incidence rate of 17 cases per million individuals (1).

Correspondence to: Dr Qiang Shu, Department of Endoscopic Surgery Center, Children's Hospital, Zhejiang University School of Medicine, 3333 Bin Sheng Road, Hangzhou, Zhejiang 310000, P.R. China

E-mail: qiangshu611@sina.com

Key words: gastric duplication, diagnosis, laparoscopy, laparotomy
The manifestation of gastric duplication varies in children with different ages, and patients may present abdominal pain, vomiting, bloody stools, feeding difficulties or other nonspecific gastrointestinal symptoms (2-4). The preoperative diagnosis of gastric duplication is difficult, and misdiagnosis according to the detection of abdominal cystic masses and ultrasonic examination findings is common.

The present study reports the cases of four gastric duplication pediatric patients admitted to the Children's Hospital of Zhejiang University between March 2010 and March 2015. The study included 3 females and 1 male, with an age range of 9 months to 11 years. All children were diagnosed with gastric duplication and received treatment. The study was approved by the Ethics Committee of the Children's Hospital of Zhejiang University School of Medicine (Hangzhou, China), and informed consent was obtained from the patients' parents. The clinical characteristics of the four cases are shown in Table I.

\section{Case reports}

Case 1. An 8-year-old female child presented with abdominal pain and vomiting that persisted for 5 days, and was admitted to the Children's Hospital of Zhejiang University. Ultrasonography examination and contrast-enhanced CT confirmed an upper abdominal cystic space-occupying lesion, which was $6 \times 3 \times 2.5 \mathrm{~cm}$ in size and was located between the pancreatic tail and stomach cavity. A diagnosis of gastric repeat deformity or cystic teratoma was initially considered following a CT scan (Fig. 1A). Laparoscopic exploration was performed the following day, in order to confirm the size of pathological changes and the degree of adhesion with the surrounding inflammatory tissues, in addition to the positional association of lesions with the stomach, and to determine the appropriate further surgical methods. During surgery, it was confirmed that the lesion was a cystic gastric duplication deformity without communication with gastric cavity. Laparoscopy-assisted resection by pulling out the duplicated gastric through the navel, and repair of the stomach was conducted in the same procedure, with the mass presented in Fig. 1B. The gastric tubes were removed at 1 day after surgery, while the patient defecated 2 days after surgery and was discharged at postoperative day 9. No incision infection, intestinal obstruction, bleeding or other gastrointestinal complications, such as leakage, occurred in this patient. 
Furthermore, no complications were reported during follow-up examinations conducted at 1 month, 1 year and 3 years. The preoperative symptoms disappeared completely, and the growth and development of the child was normal.

Case 2. A 20-month-old female child presented with an upper abdominal cystic mass that was detected by ultrasonography examination following birth. Although the mass gradually increased, no other clinical symptoms were identified during the one-year outpatient follow-up examination. When the pediatric patient was admitted to the Children's Hospital of Zhejiang University due to an abdominal mass, the cystic mass was approximately $3.5 \times 3.2 \times 2.5 \mathrm{~cm}$ in size and was located below the spleen on the left side of the abdomen, demonstrated by CT scanning (Fig. 2). A left upper abdominal cystic lesion, which possibly originated from the posterior peritoneum, was identified by CT scanning, and pancreatic pseudocyst was considered as a possible diagnosis by ultrasonography examination. Laparoscopic exploration was conducted 2 days later. The mass was located at the posterior gastric wall near the lesser curvature of the stomach, and the surrounding inflammatory adhesion was severe, thus laparotomy was performed. At 1 day after surgery, the gastric tubes were removed. The patient defecated at 2 days following surgery and was discharged at 9 days post-operation. No incision infection, intestinal obstruction, bleeding or other gastrointestinal complications, such as leakage, occurred. During the 1-month, 1-year and 3-year follow-ups, no complications were reported. All preoperative symptoms disappeared, and the child exhibited normal growth and development.

Case 3. An 11-year-old male child with intermittent abdominal pain, which persisted for 3 months prior to gastroscopic discovery of a mass in the gastric antrum, was admitted into the Children's Hospital of Zhejiang University. A $1.5-\mathrm{cm}$ submucous eminent lesion with smooth surface was discovered by gastroscopy, and the diagnosis of mucosa lesions at the gastric antrum was considered. CT examination identified a $1.2 \times 1.7 \times 1.0-\mathrm{cm}$ nodular soft tissue mass with uniform density at the distal stomach, thus the presence of a gastric stromal tumor was considered (Fig. 3A). Laparoscopic exploration was then conducted the following day. During the surgical procedure, the mass was confirmed to be a cystic gastric duplication deformity without traffic with gastric cavity. In this case, a total laparoscopic resection was conducted. Since rapid intraoperative pathological diagnosis did not suggest that the lesion was malignant, simple excision of the tumor was performed. Postoperative pathological analysis of the resected tissue was consistent with gastric duplication (Fig. 3B). The gastric tubes were removed at day 1 after surgery, while the patient defecated on day 2 and was discharged at 7 days post-surgery. No incision infection, intestinal obstruction, bleeding or other gastrointestinal complications, such as leakage, were reported. Upon follow up at 1 month, 1 and 3 years after surgery, no complications were reported. The preoperative symptoms disappeared completely, and the growth and development of the child was normal.

Case 4. A 9-month-old female child presented with melena persisting for 3 days prior to ultrasonography diagnosis of

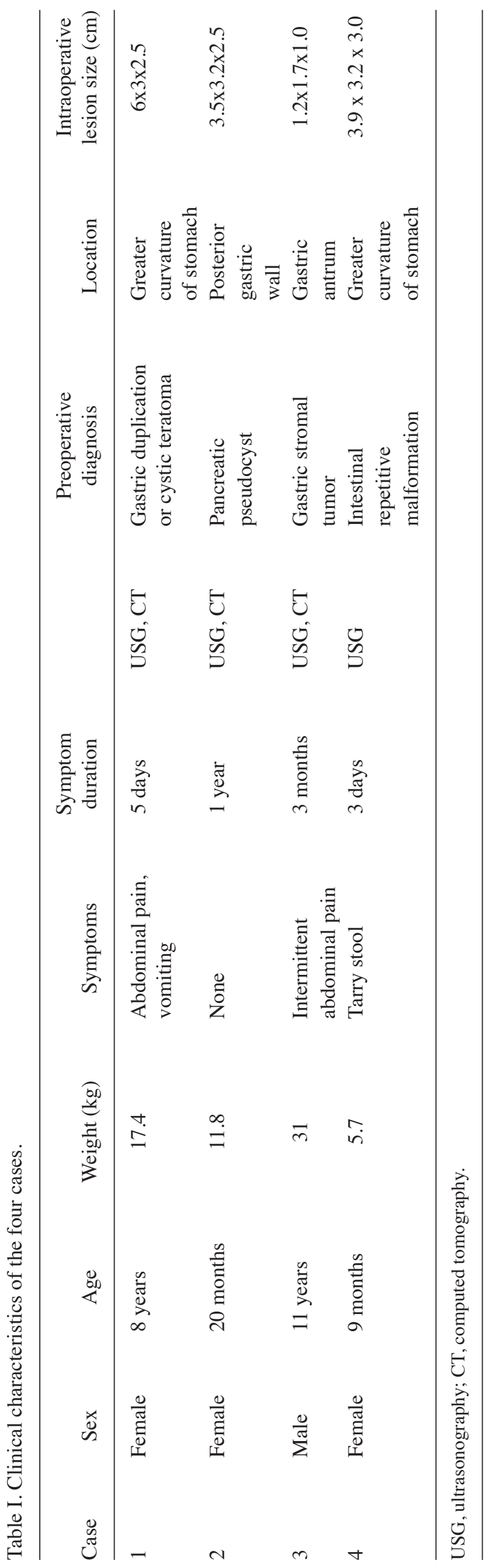



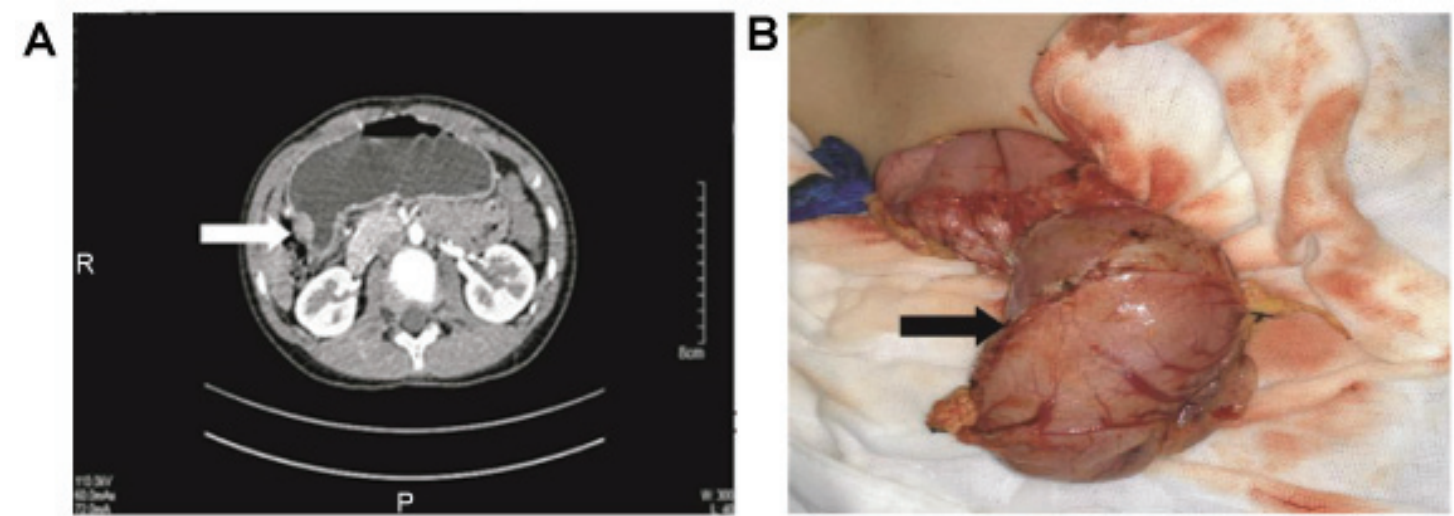

Figure 1. (A) Contrast-enhanced computed tomography demonstrated an upper abdominal cystic space-occupying lesion (arrow) in case 1. (B) Mass (indicated by the arrow) resected by laparoscopic-assisted resection by pulling out through the navel in case 1 . The size of the mass was approximately $6.0 x 3.0 x 2.5 \mathrm{~cm}$, and the wall was complete.

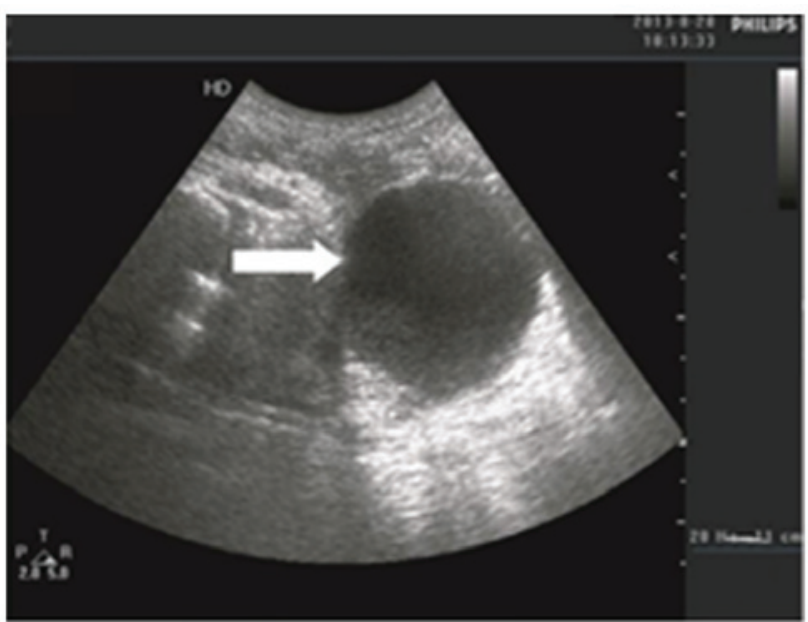

Figure 2. Computed tomography indicated a left upper abdominal cystic lesion (arrow) in case 2 .

intussusception, and was admitted to the Children's Hospital of Zhejiang University. However, air enema excluded intussusception and a right upper abdominal mass was suspected. A second ultrasonography examination identified an abdominal cystic mass, which was approximately $3.9 \times 3.2 \times 3.0 \mathrm{~cm}$ in size with a $0.4-\mathrm{cm}$ cyst wall thickness. Next, intestinal repetitive malformation was considered (Fig. 4A). During surgery, the mass was confirmed to be a cystic gastric duplication deformity without communication with gastric cavity. Thus, laparoscopy-assisted resection was conducted resection by pulling out it through the navel, as well as surgical repair of the stomach. In this case, the repeated gastric mass had adhered and corroded the transverse colon, forming a fistula. The gastric mass attached to the transverse intestines was removed along with the fistula (Fig. 4B and C). The postoperative pathology was consistent with the diagnosis of gastric duplication. Transverse colon excision was performed, thus the gastric tube was removed at 2 days post surgery. The patient defecated at 2 days after surgery and was discharged on postoperative day 10. No incision infection, intestinal obstruction, bleeding or other gastrointestinal complications (such as leakage) occurred. During follow-up at 1 month, 1 and 3 years after surgery, no complications were reported. The preoperative symptoms disappeared completely, and the child presented normal growth and development.

\section{Discussion}

Gastrointestinal tract duplications are rare congenital abnormalities that may occur anywhere along the gastrointestinal tract, from the mouth to the anus. Almost half of these abnormalities occur in the small intestine, particularly in the ileum. Gastric duplication accounts for 2-8\% of all gastrointestinal tract duplication deformities (5). The first study to report that digestive tract abnormalities are considered when digestive tract smooth muscle and gastrointestinal epithelial tissue are present in the mass, and the mass is attached to the digestive tract was completed by Rowling (6). Gastric duplication is known to be more prevalent in females (6), however, the etiology for this prevalence is unclear to date. The etiology includes anomalies occurring in the early development of fetal intestinal diverticulitis, vacuolization, caudal duplication and split notochord syndrome $(7,8)$. Gastric duplication is divided into the tube and the cyst types. The tube type of this condition is interlinked with the stomach, whereas the majority of cyst-type cases occur at the greater curvature of the stomach. Since the cyst-type of gastric duplication is not interlinked with the stomach, it is not easily diagnosed by gastrointestinal angiography and gastroscopy. Furthermore, while ultrasound, $\mathrm{CT}$ and magnetic resonance imaging are helpful for the diagnosis, confirming the diagnosis using these methods remains challenging (9).

In the present study, four cases (including 3 females and 1 male) of pediatric patients were included. Laparoscopic exploration was initially performed in all cases, and all subsequent surgical procedures were successful. The pathological type of all the masses was single cyst, which was not connected with the stomach cavity. The mass in three cases was located in the greater curvature of the stomach, while it was located in the posterior gastric wall and lesser curvature in one case. The data of the present study are consistent with previous studies on the pathology and epidemiological characteristics of this disease (5-8). Gastric duplication can be complicated with other malformations, including intestinal atresia and spinal 

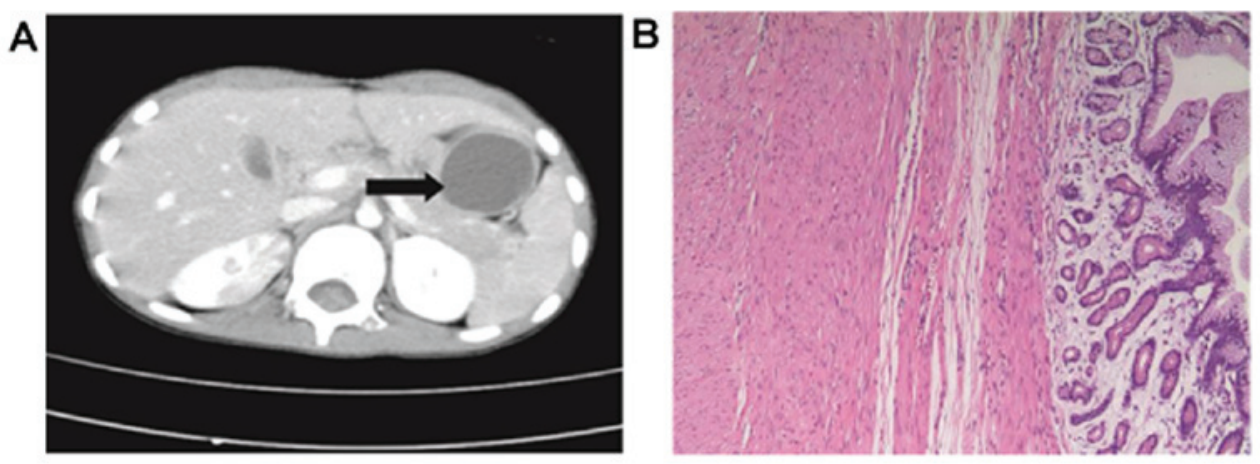

Figure 3. (A) Computed tomography scanning demonstrated a 1.2x1.7x1.0-cm nodular soft tissue lesion with uniform density at the distal stomach (arrow), and gastric stromal tumor was considered in case 3. (B) Hematoxylin and eosin staining of gastric body gland mucosal under x 50 magnification. The intramucosal congestion was evident, part of the epithelial tissue was eroded and the complete submucosal smooth muscle layer was visible in case 3.

A

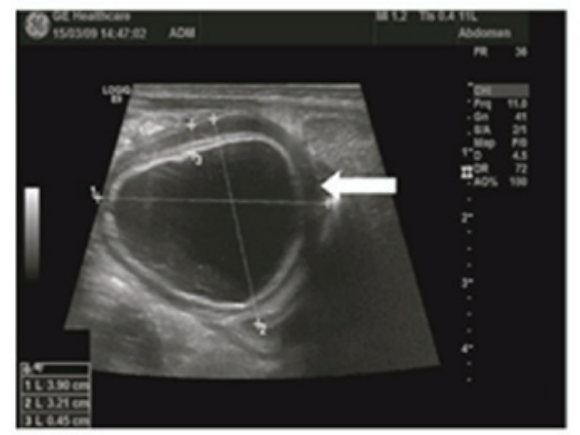

C

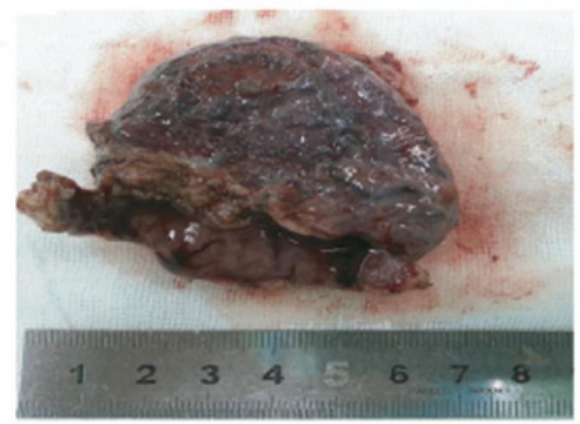

B

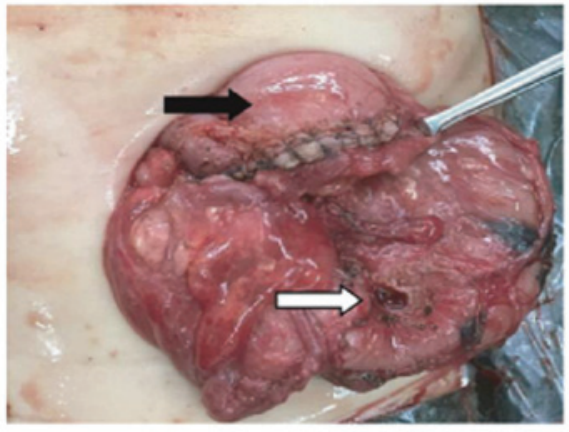

Figure 4. (A) Ultrasonography examination identified an abdominal cystic mass (arrow) in case 4, which was $3.9 \times 3.2 \times 3.0 \mathrm{~cm}$ in size with a $0.4 \mathrm{~cm}$ cyst wall. (B) Surgical repair of the stomach (black arrow). The repeated gastric mass adhered to and corroded the transverse colon, and a fistula was formed (white arrow). Intestinal perforation was visible when cut off. (C) The gross specimen was $4.5 \times 3.0 \times 3.0 \mathrm{~cm}$ in size, and was a single cyst gastric duplication deformity without communication with gastric cavity.

deformity (10), however such complications were not observed in the cases reported in present study.

Gastric duplication cysts may present with various abdominal symptoms, including a palpable abdominal mass, pain, vomiting, bloody stools, feeding difficulties and weight loss. However, all these symptoms are nonspecific. In addition, gastric duplication can also cause intestinal bleeding, adhesive obstruction and perforation, which are caused by the mucosa corrosion effect from ectopic gastric acid secretion $(11,12)$. In case 4 of the current study, the repeated gastric mass adhered to and corroded the transverse colon, and a fistula was formed, resulting in bloody stool and melena. Typically, when abdominal masses were detected by ultrasonography in the right upper abdomen, an intestinal duplication deformity is considered. Abdominal enhanced CT may demonstrate that the strengthened focal cystic wall was very close to the gastric body. This deformity can be easily misdiagnosed as gastric or pancreatic pseudocyst (13). For smaller gastric duplication deformities, the lumen of the cyst is not clear. It may thus be misdiagnosed as a gastric stromal tumor, since strengthened focal cystic wall appears in the abdominal enhanced CT scan. In the present study, case 3 was considered to be a gastric stromal tumor, and was correctly diagnosed as a duplication deformity by intraoperative pathological confirmation. Technetium 99 imaging can also confirm ectopic gastric mucosa tissue within the cyst (1). In the current study, ultrasound and contrast-enhanced CT examinations were performed in all four cases. However, the CT findings suggested gastric duplication defects only in case 1 , possibly due to the low morbidity of this disease and the inspectors' experience. Therefore, gastric 
duplication should be considered in cases presenting with the aforementioned clinical symptoms and auxiliary examination findings, including ultrasound or CT, indicating a cyst in upper abdominal cavity, particularly in the stomach area, upper pancreas and even within the chest cavity (14).

Gastrointestinal duplication deformity can lead to severe complications, thus, surgical resection treatment must be conducted once the disease is confirmed (15). Surgical resection is the only possible treatment method, and the surgical approach should be selected according to the case characteristics. Laparoscopic cyst removal is suitable for a simple cyst without severe inflammatory adhesion. However, laparoscopy-assisted resection by pulling out it through the navel is appropriate for cysts with severe inflammatory adhesion. Alternatively, laparotomy is the only choice. In the cases reported in the present study, two patients (case 1 and case 4) received laparoscopy-assisted resection by pulling out it through the navel, one patient (case 2) received total laparoscopic resection, and one patient (case 3) received laparoscopy and laparotomy. In case 4 , the repeated gastric mass adhered, corroded the transverse colon and formed a fistula; therefore the adhesion part of the transverse intestines was removed along with the gastric mass by laparoscopy-assisted resection by pulling out it through the navel. In case 1 , the cyst was smaller and a gastric stromal tumor was considered by CT, however, rapid intraoperative pathological diagnosis excluded this diagnosis, and thus simple excision of the tumor by total laparoscopic resection was performed. In case 2 , the surgical procedure was transited to laparotomy due to the following reasons: The cyst was located at the posterior gastric wall near the lesser curvature of the stomach and the surrounded inflammatory adhesion was severe; bleeding was hard to avoid; exposure was difficult and pulling it out was not possible. Complete resection without partial gastrectomy was performed in all cases, along with seromuscular layer repair.

The majority of gastric duplication deformities in children are benign, with the exception of one case of gastric adenocarcinoma reported by Min et al (16). Gastric duplication associated with ectopic pancreas tissue is common in children, and there were two cases (cases 1 and 4) with ectopic pancreatic tissue in the present study. The associated mechanism of malignant transformation of gastric duplication cyst remains unclear, thus further investigation is required in future studies.

In conclusion, pediatric gastric duplication deformity is rare and difficult to diagnose. For cases with nonspecific clinical symptoms and auxiliary examination, including ultrasound or CT scans, in which cystic mass in upper abdominal cavity is observed, particularly in the stomach area, upper pancreas and around the spleen, gastric duplication should be considered. Surgical resection is the only method of treatment for duplications. The disease in adult is prone to malignant transformation, and therefore surgical intervention is generally performed as early as possible. Laparoscopy-assisted or total laparoscopic resection is a suitable choice for uncomplicated gastric duplication deformities, and simple excision can achieve a better therapeutic effect for such deformities.

\section{Acknowledgements}

The current study was financially supported by the Zhejiang Provincial Bureau of Traditional Chinese Medicine (grant no. 2011ZA068), and the Science and Technology Agency of Zhejiang Province (grant no. 2012C13024-1).

\section{References}

1. Bonacci JL and Schlatter MG: Gastric duplication cyst: A unique presentation. J Pediatr Surg 43: 1203-1205, 2008.

2. Srivastava P, Gangopadhyay AN, Kumar V, Upadhyaya VD, Sharma SP, Jaiman R and Hasan Z: Noncommunicating isolated enteric duplication cyst in childhood. J Pediatr Surg 44: e9-e10, 2009.

3. Sakamoto K, Hasegawa S, Yamazaki Y, Makino T, Suda T and Imada T: Ileal duplication presenting as perforation: Report of a case. Surg Today 30: 445-447, 2000.

4. Okoro PE and Obiorah C: Duplication cyst of the bowel causing ileal volvulus. A case report. Niger J Med 19: 230-232, 2010.

5. Prinsloo H, Loveland J, Grieve A, Andronikou S and Valli Omar M: Gastric duplication cysts as a rare cause of haematemesis: Diagnostic challenges in two children. Pediatr Surg Int 27: 1127-1130, 2011.

6. Rowling JT: Some observations on gastric cysts. Br J Surg 46: 441-445, 1959.

7. Okamoto T, Takamizawa S, Yokoi A, Satoh S and Nishijima E: Completely isolated alimentary tract duplication in a neonate. Pediatr Surg Int 24: 1145-1147, 2008.

8. Gorsler CM, Rodriguez R, Kistler W and Baumgartner G: Coincidence of aductal pancreatic cyst and a gastric duplication cyst: A case report. Eur J Pediatr Surg 18: 123-125, 2008

9. Zhentong Feng and Long Li: The clinical application of laparoscopic diagnosis and treatment of pediatric gastrointestinal repeated deformity. Chin J Minim Invasive Surg 8: 798-799, 2008.

10. Zhang J, Zhang KR, Bai YZ and Wang W: Thoracoabdominal foregut duplication cyst with respiratory epithelium and alimentary epithelium. Am J Surg 199: e54-e56, 2010.

11. Karnak I, Ocal T, Senocak ME, Tanyel FC and Büyukpamükcu N: Alimentary tract duplications in children: Report of 26 years experience. Turk J Pediatr 42: 118-125, 2000.

12. Schalamon J, Schleef J and Höllwarth ME: Experience with gastro-intestinal duplications in childhood. Langenbeck's Arch Surg 385: 402-405, 2000.

13. Faerber EN, Balsara R, Vinocur CD and De Chadareveian JP: Gastric duplication with hemoptysis: CT findings. AJR Am J Roentgenol 161: 1245-1246, 1993.

14. Xiaojuan Wu, Xiaogui Pan and Honggang Liu: Pediatric diagnosis and treatment of huge gastric repeat deformity analysis and follow-up of a case. The Chin J Pediatr Surg 31: 155, 2010.

15. Yang DM, Kim HC, Choi SI and Lim SJ: Sonographic diagnosis of gastric duplication cyst communicating with the gastric lumen. J Clin Uhrasound 39: 550-552, 2011.

16. Min SH, Kim HY, Kim SH, Jung SE, Park KW, Kim WS and Park SH: Gastric adenomyoma mimicking gastric duplication cyst in a 5-year-old girl. J Pediatr Surg 47: 1019-1022, 2012. 